Research Article

\title{
Impact Angle Control Guidance to Intercept Moving Targets by Virtual Target Technique
}

\author{
Libing Hou $\mathbb{D}$, Jihong Zhu $\mathbb{D}^{\mathbb{B}}$, Minchi Kuang $\mathbb{D}$, and Heng Shi \\ Tsinghua University, 100084 Beijing, China \\ Correspondence should be addressed to Jihong Zhu; jihong_zhu@hotmail.com
}

Received 10 May 2021; Accepted 26 November 2021; Published 21 December 2021

Academic Editor: Teng Wu

Copyright ( 2021 Libing Hou et al. This is an open access article distributed under the Creative Commons Attribution License, which permits unrestricted use, distribution, and reproduction in any medium, provided the original work is properly cited.

\begin{abstract}
To solve the problem regarding the impact angle of the missile, this paper proposes a novel guidance law, which can control the missile to hit the target at the desired angle. The key of the guidance law is selecting a moving point on the collision line as the virtual target, and the tactical requirements can be fulfilled by the missile directly pursuing the virtual target. The Lyapunov stable theory is used to prove the convergence of the proposed guidance law. The guidance command is generated by a PID controller to make the missile towards the virtual target. The proposed guidance law makes the lateral acceleration of the missile converge to zero, which leads the angle of attack to zero, and it theoretically guarantees the flight path angle equals the attitude angle. Numerical simulations demonstrate this impact angle control guidance law is very accurate and robust. Regardless of whether the initial heading error is large or small, the missile which employs the proposed guidance law can always hit the target from the preset direction and the guidance process is smooth.
\end{abstract}

\section{Introduction}

Guidance laws aim to achieve desired impact angle, as well as zero miss distance, are pushed forward by a number of techniques. Literature [1-7] derived guidance laws with impact angle constraint based on proportional navigation guidance (PNG). Ratnoo and Ghose [2] explored the influence of navigation gain on impact angle and designed a two-stage PNG law to achieve the desired impact angle. But this method usually provides a long trajectory, which makes it difficult to be implemented on missiles with range limit in practice. A nonswitching guidance law for trajectory shaping control was proposed by Ratnoo [3], where the lineof-sight (LOS) angle is added into the PNG law. Though this impact angle control guidance (IACG) law is capable of approaching stationary targets at all angles, it fails to achieve the angle control when the target is moving. Vairavan and Ratnoo [1] made the improvement by using a variable navigation gain, which is able to deal with the moving target, yet it is not robust enough when the target's velocity is vary- ing. These PNG-based impact angle control guidance techniques are simple in form and cost little in the calculation, but some constraints on the impact angle cannot strictly satisfied when the initial heading coincides with the LOS. The sliding mode control (SMC) technique is another commonly used technique in the field of IACG. Literatures [8-19] use SMC to achieve desired impact angle. Guidance laws based on a conventional SMC can only ensure asymptotic convergence. A guidance law based on terminal sliding mode control was proposed in [10], which satisfied the finite-time convergence. Hu et al. [16] combined SMC and virtual target strategy to control collision time and angle. Guidance laws based on SMC have strong robustness, which are even effective on maneuvering targets. However, the discrete terms in the guidance formula always cause inevitable oscillations in the guidance commands, which is not suitable for actual implementations. The SMC technique was introduced to design a guidance law that makes multiple missiles hit the target in a preset direction at the same time [18], and it utilized range-to-go and radial relative velocity rather than 
time-to-go to construct the sliding surface to avoid performance degradation. Khankalantary and Sheikholeslam combined SMC, backstepping, and super-twist extend state observer to propose a robust guidance law [19] and verified its performance by a dynamics model. In addition to PNG and SMC, Ryoo et al. [20] proposed the IACG method to calculate the remaining time, and it succeeded in interception stationary targets at all angles. But it also failed against moving target. A trajectory shaping method based on Archimedean spiral was proposed in [21] to deal with the terminal angle constraint. But sometimes the problem cannot be solved since the calculated Archimedean spiral may be a bad curve. Yan et al. [22] introduced a IACG law based on computational geometry. This trajectory formed by this approach is composed of a log-aesthetic space curve segment and a collision line segment, and the curvature can be adjusted to satisfy the impact angle constraint. Despite these geometry-based methods are sufficient to produce desired trajectories, the amount of real-time calculation is relatively large, which makes it difficult to be implemented on a standard missile's on-board computer. Moreover, $\mathrm{Hu}$ et al., Chen et al., and Shi et al. $[16,23,24]$ used the virtual target method in the design of guidance laws. Chen et al. [23] took a virtual target on the reference trajectory, enabling the unmanned aerial vehicle to move along the reference trajectory. The key of this strategy is calculation of the speed of the virtual target. But this method cannot ensure the convergence within a finite time. In literature [16], the virtual target was utilized to optimize the impact time, and angle constraint is finished by SMC. Shi et al. proposed a predictive guidance law [24], the virtual target is the predictive intercept point, which is based on the target's velocity and the time to go, and the missile pursues the virtual target by PNG law. A suboptimal IACG law based on finite-time state-dependent Riccati equation was proposed for an interceptor to pursue an aerial vehicle in a 3D engagement [25]. Kim et al. proposed a IACG law [26] for nose-dive missile which can maximize its terminal velocity, but the assumption that the initial angle is close to vertical is unreasonable.

To overcome the shortcomings of the aforementioned methods, this paper is aimed at proposing a novel IACG strategy based on the virtual target approach to support the practical application. To this end, a virtual target is designed firstly via geometry analysis considering the impact angle constraint. The interceptor is then controlled to follow the virtual target. The guidance command is generated based on the error of the missile's flight path angle and the LOS (the missile and the virtual target) angle. The convergence of the proposed method is proved analytically via Lyapunov stability theory, and the geometric conditions for convergence are derived. Numerical simulations with comparisons confirm the effectiveness and feasibility of the proposed strategy.

The contributions of this paper are as follows:

(i) This IACG law achieves the desired impact angle with less calculation

(ii) The guidance process is smooth with no chattering

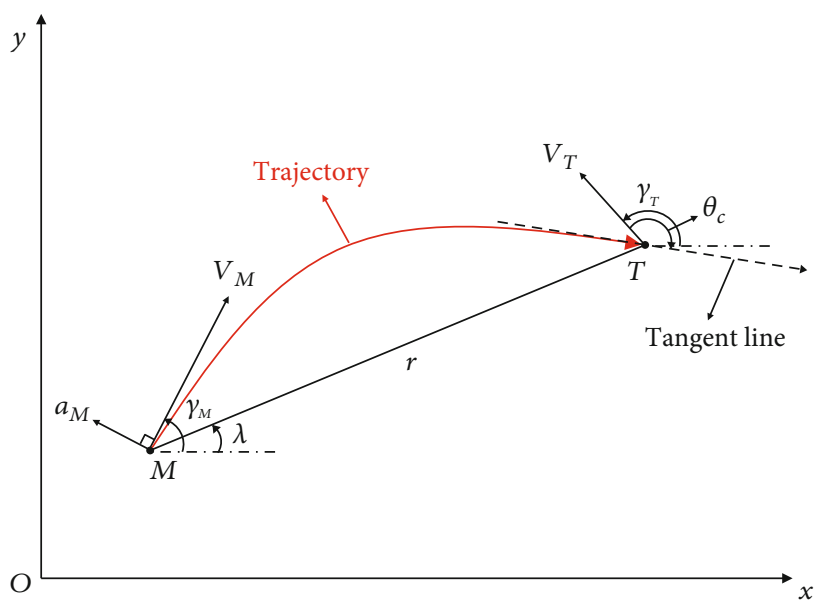

FIGURE 1: Missile-target engagement.

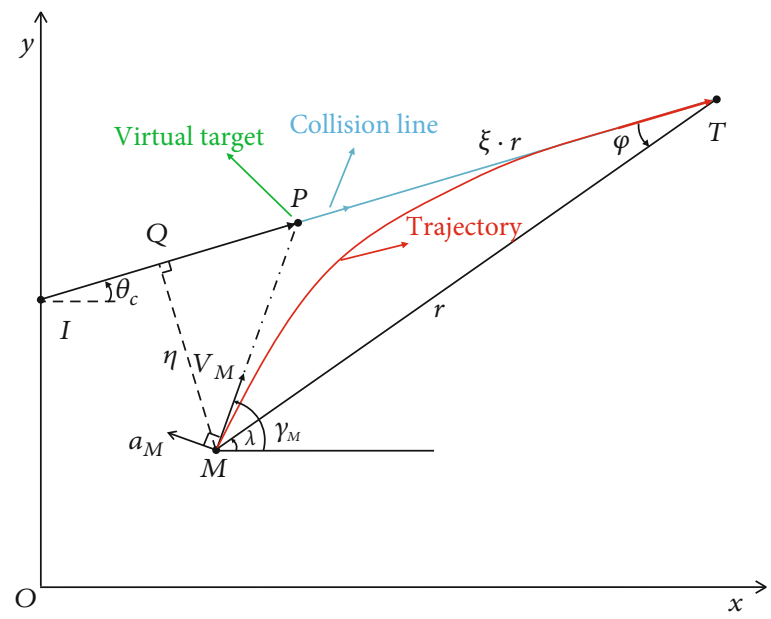

FIGURE 2: Stationary target.

(iii) The lateral acceleration of the missile converges to zero, which leads to the angle of attack to zero, and it theoretically guarantees the trajectory angle can represent the attitude angle

The rest of the paper is organized as follows. Section 2 presents the formulation of the impact angle control engagement mathematical model, followed by the derivation of the impact angle control guidance law in Section 3. Numerical simulations are presented in Section 4.

\section{Problem Formulation}

Consider a planar homing engagement scenario of a missile against a moving target, as shown in Figure 1. $M$ and $T$ denote the missile and the target, and their velocities are $V_{M}$ and $V_{T}$, and $\gamma_{M}$ and $\gamma_{T}$ represent their flight path angles, respectively. $r$ stands for the distance between the missile and the target. The angle of line-of-sight is denoted by $\lambda$, and $a_{M}$ refers to the lateral acceleration of the missile. $\theta_{c}$ is the desired impact angle. The engagement kinematics 


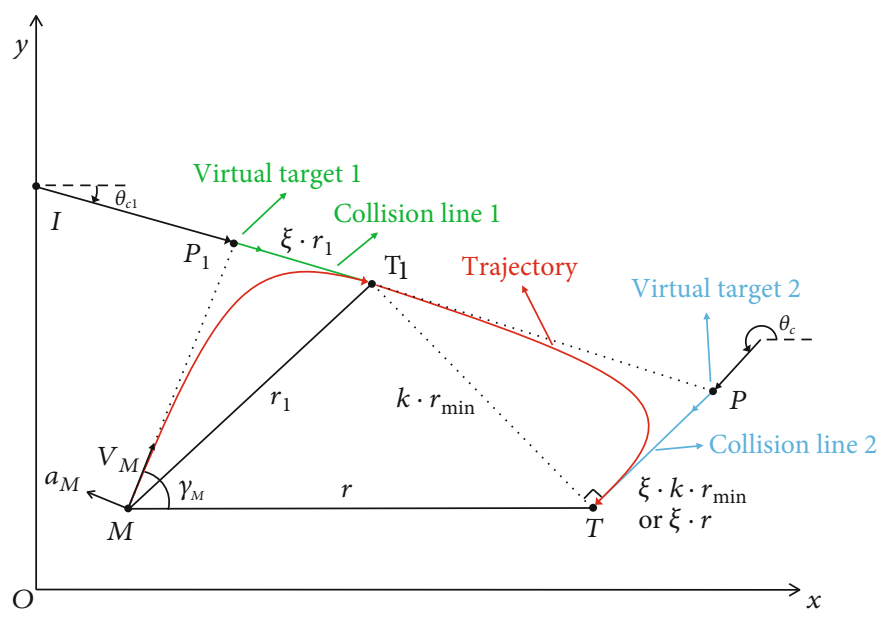

Figure 3: Omnidirectional attack.

for this problem can be given as follows:

$$
\left\{\begin{array}{l}
\dot{x}_{M}=V_{M} \cos \gamma_{M}, \\
\dot{y}_{M}=V_{M} \sin \gamma_{M}, \\
\dot{\gamma}_{M}=\frac{a_{M}}{V_{M}}, \\
\dot{x}_{T}=V_{T} \cos \gamma_{T}, \\
\dot{y}_{T}=V_{T} \sin \gamma_{T}, \\
r=\sqrt{\left(x_{T}-x_{M}\right)^{2}+\left(y_{T}-y_{M}\right)^{2}}, \\
\dot{r}=V_{T} \cos \left(\gamma_{T}-\lambda\right)-V_{M} \cos \left(\gamma_{M}-\lambda\right), \\
\dot{\lambda}=\frac{V_{T} \sin \left(\gamma_{T}-\lambda\right)-V_{M} \sin \left(\gamma_{M}-\lambda\right)}{r} .
\end{array}\right.
$$

Assuming $t_{f}$ is the stop time of this engagement, and the missions of the paper can be described as the following objectives:

$$
\left\{\begin{array}{l}
r\left(t_{f}\right)=0, \\
\gamma_{M}\left(t_{f}\right)-\gamma_{T}\left(t_{f}\right)=\theta_{c}, \\
a_{M}\left(t_{f}\right) \longrightarrow 0 .
\end{array}\right.
$$

If the target is stationary, its flight path angle is considered equal to 0 . In the sequel, the line segment between $A$ and $B$ and its length are denoted by $A B$ and $|A B|$, respectively. To facilitate the analysis of this engagement, assumptions are made as follows:

Assumption 1. The velocities of the missile and the target are constant and available.

Assumption 2. The autopilot dynamics is much faster than the missile dynamics.
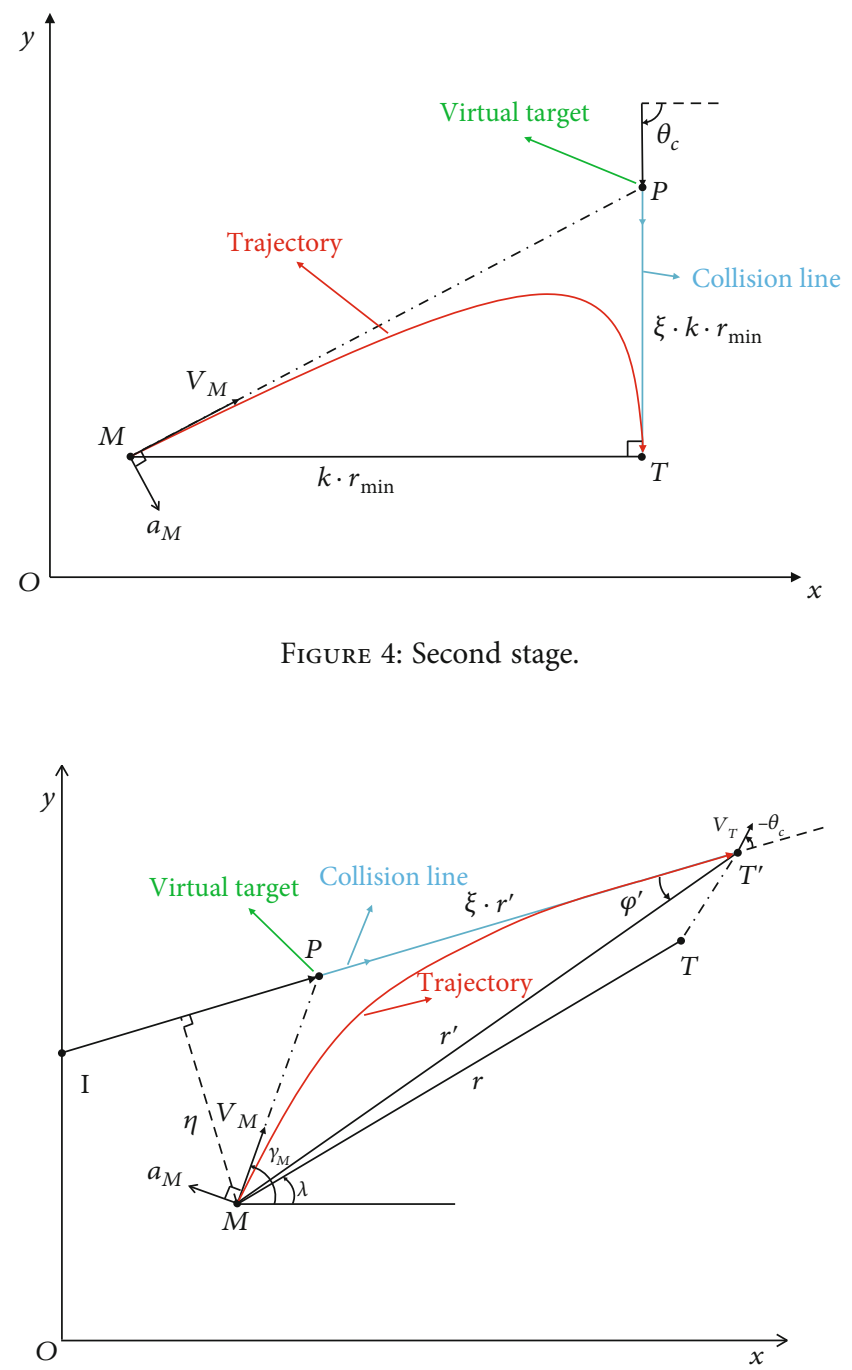

Figure 5: Moving target. 
TABLE 1: $\left|T T_{1}\right|$ 's influence on interception result.

\begin{tabular}{lcccccc}
\hline$k$ & 1 & 2 & 3 & 4 & 5 & 6 \\
\hline Impact angle (deg) & -20.167 & -49.102 & -64.691 & -77.950 & -91.4516 & -89.997 \\
Miss distance (m) & 59.027 & 60.323 & 46.409 & 26.309 & 5.310 & 0.4985 \\
\hline
\end{tabular}

\section{Virtual Target Method for Impact Angle Control}

In this section, a virtual target method is proposed to control the impact angle and eliminate miss distance. The key of this method is selecting a moving point from the collision line as the virtual target, and it approaches the real target along the collision line. The missile pursues the virtual target by pure pursuit guidance law, and it will first reach the collision line and then hit the target at the desired angle.

3.1. Design of Guidance Law with Impact Angle Control. In this subsection, the virtual target method is designed to make the missile hit the target from the desired direction. As is shown in Figure 2, the target is stationary. The line from $I$ to $T$ represents the collision line, which is denoted by $I T$, and its angle is $\theta_{c}$ (the desired impact angle). $\eta$ is the distance from $M$ to line IT. The virtual target is a moving point on the collision line, and it is denoted by $P . \mid P T$ $\mid=\xi r$, where $\xi \in(0,1)$, so $\left|\gamma_{M}-\lambda\right|<90^{\circ} . \varphi$ is the angle between line $P T$ and line $M T$. Assuming $\varphi$ is less than $90^{\circ}$ and $V_{M}$ always points to the virtual target. The missile will first reach the collision line and then move to the real target along the collision line at the desired angle. Strict proofs will be given in the next subsection.

The proposed virtual target method is strictly applicable only when $\varphi<90^{\circ}$; otherwise, $r$ may be not converged. But the two-stage guidance method can release the restricted condition. The two-stage method is shown in Figure 3.

The guidance procedure is divided into two stages by the point $T_{1}$, the guidance methods employed by the two stages are both the proposed methods above. $\theta_{c}$ and $\theta_{c 1}$ are the desired impact angle and the first-stage desired impact angle, and the missile first reaches $T_{1}$ at an angle of $\theta_{c 1}$ and then hits $T$ at the desired angle of $\theta_{c}$. The key of the two-stage guidance method is solving the length of $\left|T T_{1}\right|$ which can achieve the desired impact angle as well as the zero miss distance in the second stage. The second stage can be simplified to the engagement shown in Figure 4; the desired impact angle is $-90^{\circ}$. The algorithm to address the length of $\mid T T_{1}$ I is placed in the next subsection.

If the target is moving as shown in Figure 5, the above conclusion could be extended against a moving target. The essential of the extension is to estimate the stop time, which is denoted by $t_{f}$, and the approximation is called $t_{g o}$. It must be noted that if the target escapes at a speed of $V_{T}$ and $V_{T}>V_{M}$, the mission of the missile is likely to fail, so we assume $V_{T}<V_{M}$. As is shown in Figure 5, $V_{T}$ is the velocity of the target. Target will arrive at $T^{\prime}$ after $t_{g o}$, and $\left|T T^{\prime}\right|=V_{T} t_{g o}$. If $t_{g o}=t_{f}, T^{\prime}$ will be a stationary point. Assuming $\left|T T^{\prime}\right|$ is small, and it cannot

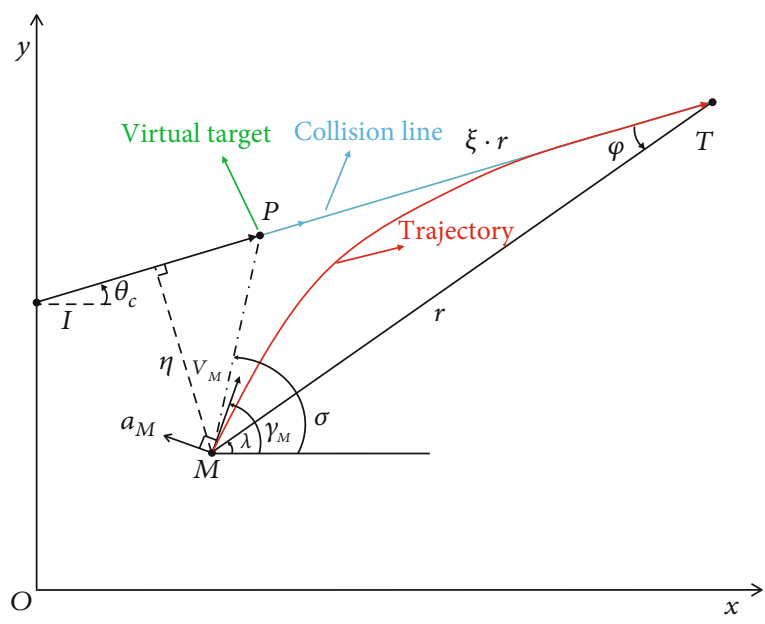

Figure 6: Actual missile target engagement.

result in $\varphi \gg 90^{\circ}$. Because $\left|T T^{\prime}\right|$ is small, $r^{\prime} \simeq r$, and their convergences are consistent. According to the conclusion for stationary targets, if $r^{\prime}$ is convergent and $T^{\prime}$ can be regarded as a stationary target, $M$ will first reach the collision line $I T^{\prime}$ and then arrive at $T^{\prime}$ with an angle of $\theta_{c}$. The next subsection will give the convergence conditions of $r^{\prime}$ and the algorithm to calculate $t_{g o}$.

3.2. Proofs of the Guidance Method. In this subsection, strict proofs of the guidance method will be provided.

\subsubsection{Stationary Target}

Proposition 3. If the missile target engagement is shown in Figure 2, the missile will reach the collision line first and then move to the real target along the collision line. The terminal lateral acceleration of the missile converges to 0 .

Proof. Referring to Figure 2,

$$
\begin{gathered}
|P T|=\xi r, \\
\xi \in(0,1), \\
\angle P M T<\angle M P T .
\end{gathered}
$$

In $\triangle P M T$, if $\angle P M T \geq 90^{\circ}$ and $\angle P M T+\angle M P T>180^{\circ}$, it breaks the theorem that the sum of the internal angles of a triangle is equal to 180 , so

$$
\angle P M T=\left|\gamma_{M}-\lambda\right|<90^{\circ} .
$$


TABle 2: Simulation conditions for Case I.

\begin{tabular}{lcccccc}
\hline$r_{T}(\mathrm{~m})$ & $V_{T 0}(\mathrm{~m} / \mathrm{s})$ & $\gamma_{T 0}(\mathrm{deg})$ & $d V_{T}\left(\mathrm{~m} / \mathrm{s}^{2}\right)$ & $r_{M}(\mathrm{~m})$ & $V_{M}(\mathrm{~m} / \mathrm{s})$ & $\gamma_{M 0}(\mathrm{deg})$ \\
\hline$(10000,0)$ & 0 & 0 & 0 & $(0,0)$ & 250 & 0 \\
$a_{M}^{\max }\left(\mathrm{m} / \mathrm{s}^{2}\right)$ & $\xi$ & $k$ & $t_{\tau}(\mathrm{s})$ & & & \\
200 & 0.6 & 10 & 0.2 & & & \\
\hline
\end{tabular}

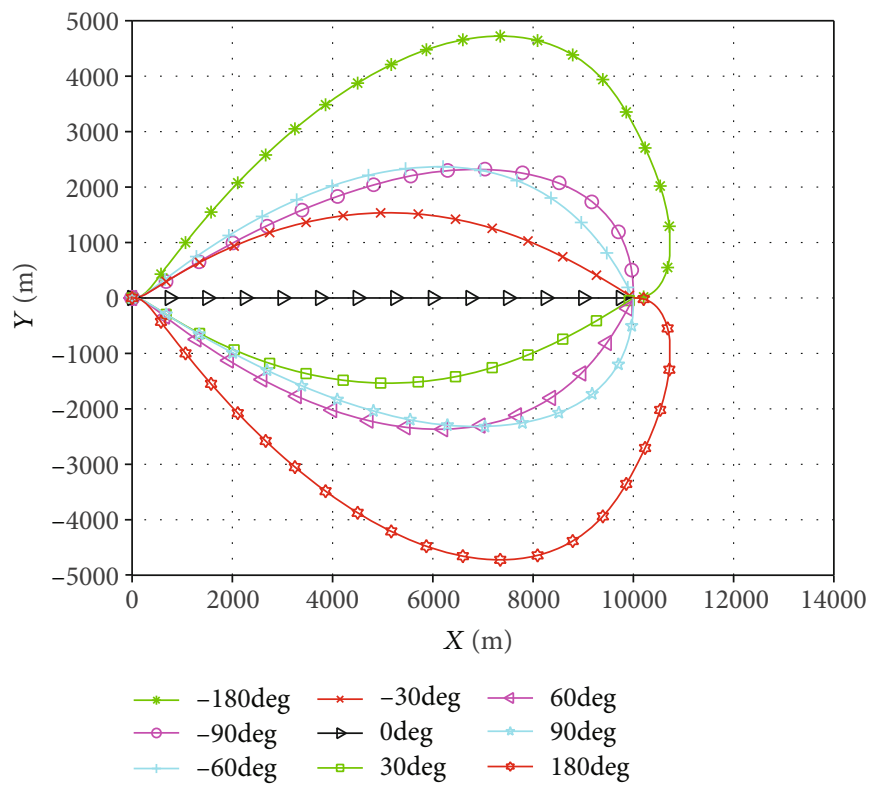

FIgURE 7: Case I: all-aspect attack.

Substituting Equation (4) to Equation (1),

$$
\dot{r}=-V_{M} \cos \left|\gamma_{M}-\lambda\right|<0 .
$$

Choose Lyapunov function as follows:

$$
V=r .
$$

Considering Equation (5),

$$
\begin{gathered}
\dot{V}=\dot{r}<0, \\
V \dot{V}<0 .
\end{gathered}
$$

According to Lyapunov stability theory, $r$ will converge to a certain value at the stop time of the engagement. If this value is not 0 , the process will continue until $r=0$. Similarly, $|P T|$ will also converge to 0 at that time. It means that the virtual target and the missile will reach the real target simultaneously.

Now considering the derivatives of $r$ and $\eta$,

$$
\dot{\eta}=-V_{M} \sin \left(\left|\gamma_{M}-\lambda\right|+\varphi\right) .
$$

Combining Equation (5) and Equation (8),

$$
\begin{aligned}
\frac{r}{|\dot{r}|} & =\frac{\eta}{V_{M} \cos \left(\left|\gamma_{M}-\lambda\right|\right) \sin \varphi} \\
& >\frac{\eta}{V_{M}\left[\sin \left(\left|\gamma_{M}-\lambda\right|\right) \cos \varphi+\cos \left(\left|\gamma_{M}-\lambda\right|\right) \sin \varphi\right]} \\
& =\frac{\eta}{V_{M} \sin \left(\left|\gamma_{M}-\lambda\right|+\varphi\right)}=\frac{\eta}{|\dot{\eta}|} .
\end{aligned}
$$

Equation (9) shows that the missile always takes more time to reach the target than it takes to reach line IT. So, we can conclude that the missile will first reach line IT, then move along the collision line with no maneuvering, and finally arrive at the real target, so the terminal lateral acceleration of the missile always converges to 0 .

Considering the two-stage guidance method shown in Figure 3, if the lateral acceleration of the missile is not limited, the missile will hit the target at the desired angle as long as $\left|T T_{1}\right|>0$, but in reality, the lateral acceleration of the missile cannot exceed its maximum, and there is a minimum turning radius $R_{\min }$, so is $\left|T T_{1}\right|$. Assuming $\mid T$ $T_{1} \mid=k R_{\min }$, it is easy to find an approximate minimum of $\left|T T_{1}\right|$ with the numerical methods. Taking $\xi=0.6$ as an example, the desired impact angle is $-90^{\circ}$. The result 
TABLE 3: Simulation result for Case I.

\begin{tabular}{|c|c|c|c|c|c|}
\hline$\overline{\theta_{c}(\operatorname{deg})}$ & -180 & -90 & -60 & -30 & 0 \\
\hline Impact angle (deg) & -180.0553 & -90.0097 & -60.0028 & -30.0010 & 0 \\
\hline Error of impact angle (deg) & 0.0553 & 0.0097 & 0.0028 & 0.0010 & 0 \\
\hline Miss distance $(\mathrm{m})$ & 0.0730 & 0.0436 & 0.1999 & 0.0045 & 0.2460 \\
\hline$\theta_{c}(\mathrm{deg})$ & 30 & 60 & 90 & 180 & \\
\hline Impact angle (deg) & 30.0010 & 60.0027 & 90.0097 & 180.0553 & \\
\hline Error of impact angle (deg) & -0.001 & -0.0027 & -0.0097 & -0.0553 & \\
\hline Miss distance $(\mathrm{m})$ & 0.0045 & 0.1999 & 0.0436 & 0.0730 & \\
\hline
\end{tabular}

TABle 4: Simulation conditions for Case II.

\begin{tabular}{lcccccc}
\hline$r_{T}(\mathrm{~m})$ & $V_{T 0}(\mathrm{~m} / \mathrm{s})$ & $\gamma_{T 0}(\mathrm{deg})$ & $d V_{T}\left(\mathrm{~m} / \mathrm{s}^{2}\right)$ & $r_{M}(\mathrm{~m})$ & $V_{M}(\mathrm{~m} / \mathrm{s})$ & 250 \\
\hline$(5000,0)$ & 0 & 0 & 0 & $(0,0)$ & \\
$a_{M}^{\max }\left(\mathrm{m} / \mathrm{s}^{2}\right)$ & $\xi$ & $t_{\tau}(\mathrm{s})$ & $\theta_{c}(\mathrm{deg})$ & & \\
200 & 0.6 & 0.2 & -90 & & \\
\hline
\end{tabular}

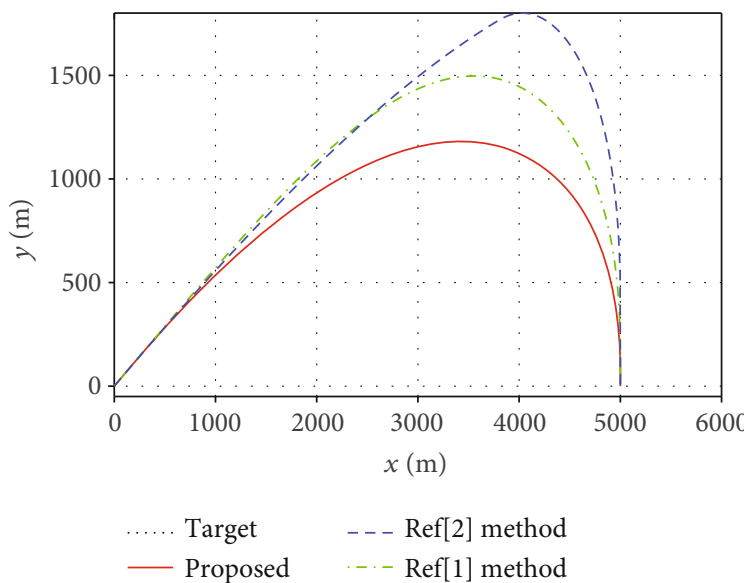

(a) Trajectories $\left(\gamma_{M 0}=30^{\circ}\right)$

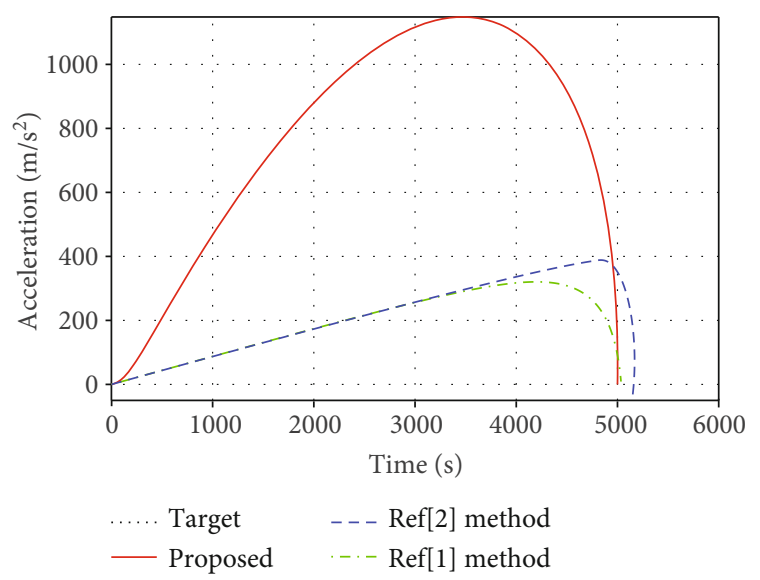

(c) Trajectory $\left(\gamma_{M 0}=5^{\circ}\right)$

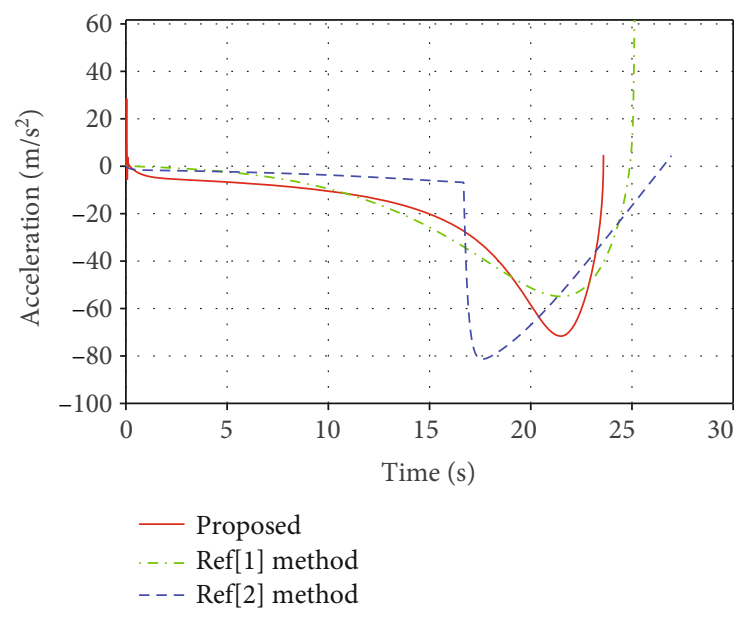

(b) Acceleration $\left(\gamma_{M 0}=30^{\circ}\right)$

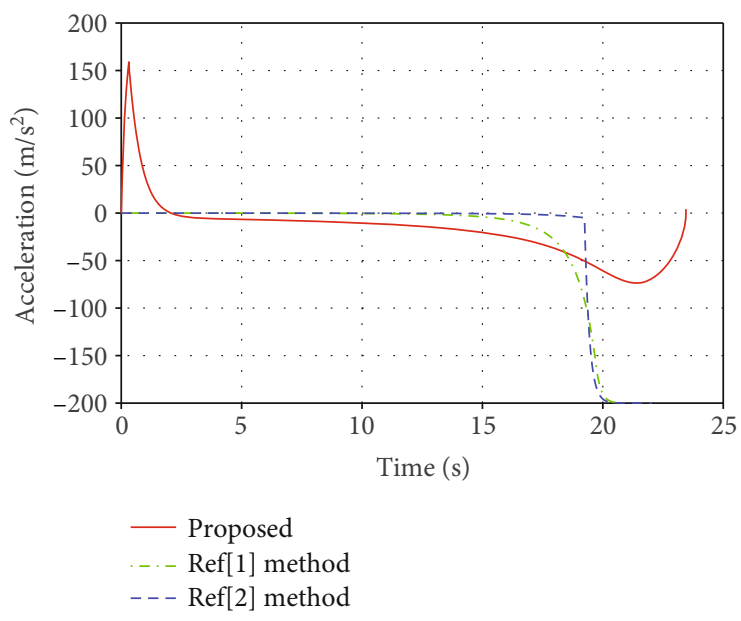

(d) Acceleration $\left(\gamma_{M 0}=5^{\circ}\right)$

FIGURE 8: Case II: stationary target. 
TABle 5: Simulation result for Case II.

(a)

\begin{tabular}{lccc}
\hline$\gamma_{M 0}=30^{\circ}$ & Proposed & Ref [2] method & Ref [1] method \\
\hline Impact angle (deg) & -90.0205 & -89.5548 & -90.6141 \\
Error of impact angle (deg) & 0.0205 & -0.4452 & 0.6141 \\
Miss distance $(\mathrm{m})$ & 0.2131 & 0.1698 & 0.1853 \\
\hline
\end{tabular}

(b)

\begin{tabular}{lccc}
\hline$\gamma_{M 0}=5^{\circ}$ & Proposed & Ref [2] method & Ref [1] method \\
\hline Impact angle (deg) & -90.0283 & -75.4084 & -117.1595 \\
Error of impact angle (deg) & 0.0283 & -14.5916 & 27.1595 \\
Miss distance $(\mathrm{m})$ & 0.0604 & 35.8176 & 151.3314 \\
\hline
\end{tabular}

TABLE 6: Simulation conditions for Case III.

\begin{tabular}{lccccc}
\hline$r_{T}(\mathrm{~m})$ & $V_{T 0}(\mathrm{~m} / \mathrm{s})$ & $\gamma_{T 0}(\mathrm{deg})$ & $d V_{T}\left(\mathrm{~m} / \mathrm{s}^{2}\right)$ & $r_{M}(\mathrm{~m})$ & $V_{M}(\mathrm{~m} / \mathrm{s})$ \\
\hline$(5000,0)$ & 50 or 100 & 0 & 0 & $(0,0)$ & 250 \\
$a_{M}^{\max }\left(\mathrm{m} / \mathrm{s}^{2}\right)$ & $\xi$ & $t_{\tau}(\mathrm{s})$ & $\theta_{c}(\mathrm{deg})$ & & \\
200 & 0.6 & 0.2 & -90 & & \\
\hline
\end{tabular}

is shown in Table $1 k_{\min }>5$. The time lag due to the missile autopilot and actuator dynamics cannot be neglected, and the value of $k$ we choose is always greater than $k_{\min }$, so making too much efforts to determine the accurate value of $k_{\min }$ is not worthy, and 8 or 10 may be a good choice.

\subsubsection{Moving Target}

Proposition 4. If missile target engagement as is shown in Figure $5,\left|T T^{\prime}\right|$ is small and $t_{g o}=t_{f}$. The convergence condition of $r$ is as follows:

$$
\xi<\sin \left(\cos ^{-1}\left(\frac{V_{T}}{V_{M}}\right)\right) .
$$

Proof. According to Lyapunov stability theory, $r^{\prime}$ will converge to 0 at the stop time of the engagement as long as $r^{\prime}$ is always less than 0 . Because $\left|T T^{\prime}\right|$ is small, $r^{\prime} \simeq r$.

$$
\begin{aligned}
r & =V_{T} \cos \left(\gamma_{T}-\lambda\right)-V_{M} \cos \left(\gamma_{M}-\lambda\right) \\
& \leq V_{T}-V_{M} \cos \left(\gamma_{M}-\lambda\right) \leq V_{T}-V_{M} \cos \left(\sin ^{-1}(\xi)\right)<0 .
\end{aligned}
$$

We can obtain the following:

$$
\xi<\sin \left(\cos ^{-1}\left(\frac{V_{T}}{V_{M}}\right)\right) .
$$

If $\xi$ satisfies Equation (12), $r$ will be shorter no matter which direction $V_{T}$ points that means $r$ will converge to 0 at the end of the engagement, so do $r^{\prime}$. Because $t_{g o}=t_{f}$,
$T^{\prime}$ can be regarded as a stationary target; according to the conclusion for stationary targets, the missile will first reach line $I T^{\prime}$ then move to $T^{\prime}$ at an angle of $\theta_{c}$ with no maneuvering.

As analyzed above, $\left|T T^{\prime}\right|$ must be small and precise to ensure the accuracy of the result, so the method to estimate $t_{f}$ is critical. Here are two algorithms to estimate $t_{f}$; they are simple enough to assure an efficient calculation.

$$
t_{g o 1}=\frac{r}{|\dot{r}|}
$$

$$
\left\{\begin{array}{l}
t_{g o 2}=\frac{r}{\delta v}, \\
\delta v=\sqrt{\left(V_{\mathrm{T}} \cos \gamma_{T}-V_{A} \cos \gamma_{A}\right)^{2}+\left(V_{T} \sin \gamma_{T}-V_{A} \sin \gamma_{A}\right)^{2}} .
\end{array}\right.
$$

Equation (13) is an algorithm to calculate $t_{\text {go }}$ used in many guidance laws, but it may be invalid in the proposed guidance method, especially when $V_{T}$ is not small, because $\dot{r}$ may be close to 0 and it could result in an infinity of $\left|T T^{\prime}\right|$, and it breaks the assumption that $\mid T$ $T^{\prime}$ । is small; consequently, $r$ may be not converged. So, we choose Equation (14) to calculate $t_{g o}$, since it not only avoids $\left|T T^{\prime}\right|$ tending to infinity but also meets the precision required for the guidance method.

3.3. Generation of Guidance Command. The above derivations are based on the assumption that $V_{M}$ always points to the virtual target. In reality, this assumption is not always satisfied. Take it as an optimization goal, and the command 


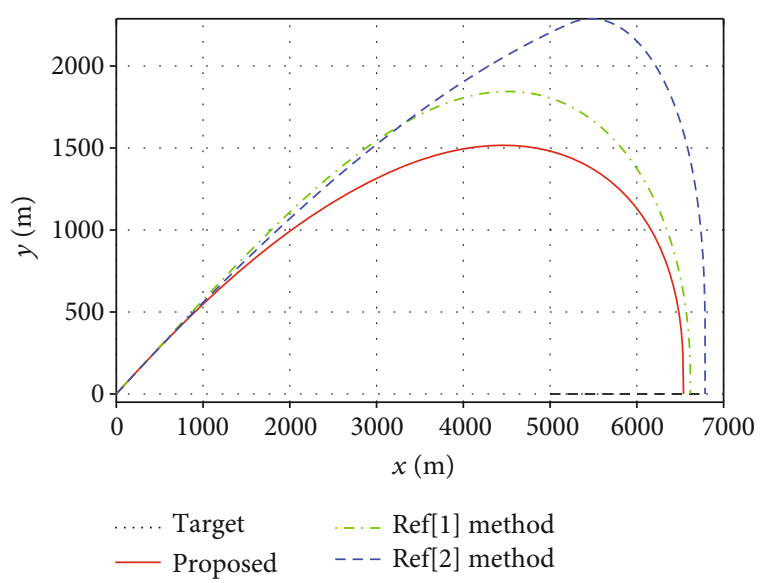

(a) Trajectories $\left(V_{T}=50 \mathrm{~m} / \mathrm{s}\right)$

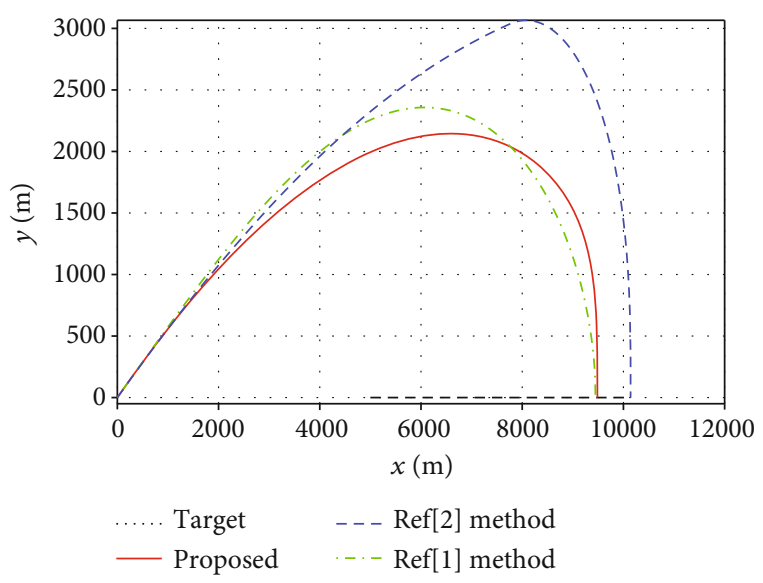

(c) Trajectories $\left(V_{T}=100 \mathrm{~m} / \mathrm{s}\right)$

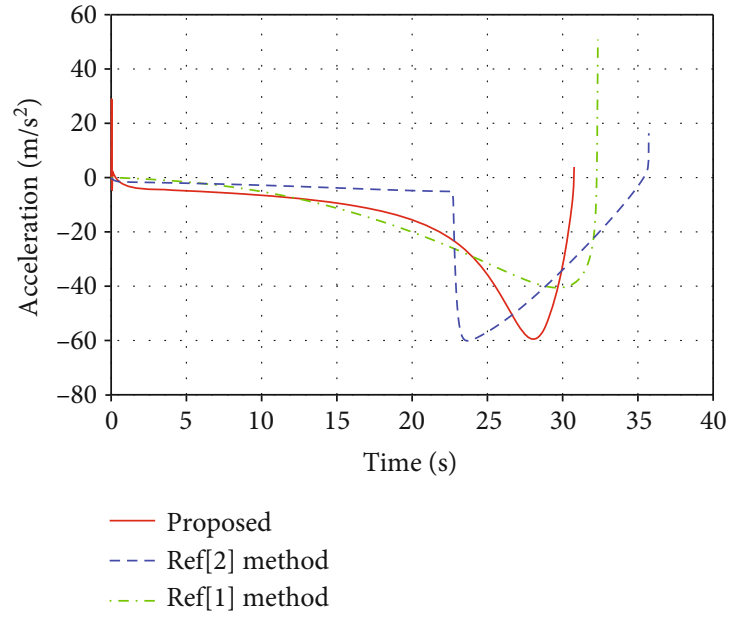

(b) Acceleration $\left(V_{T}=50 \mathrm{~m} / \mathrm{s}\right)$

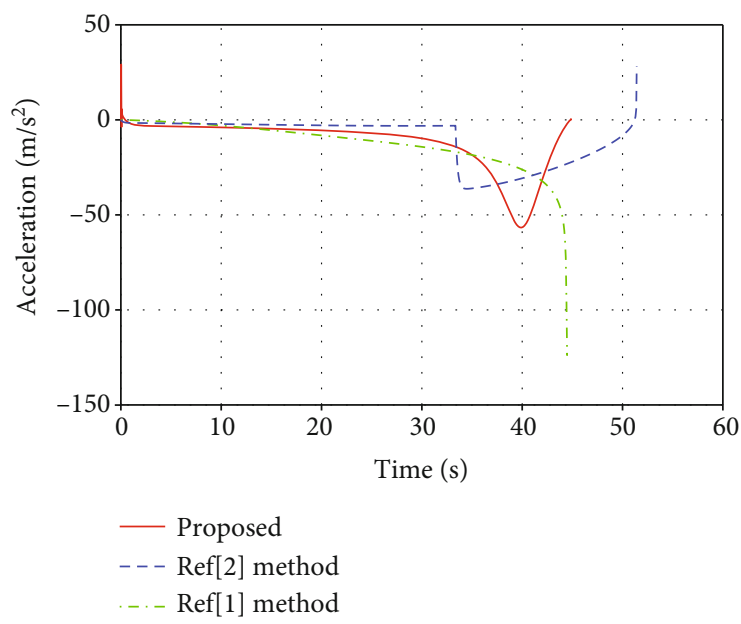

(d) Acceleration $\left(V_{T}=100 \mathrm{~m} / \mathrm{s}\right)$

FIgURE 9: Case III: constant velocity target.

TABle 7: Simulation result for Case III.

(a)

\begin{tabular}{lccc}
\hline$V_{T}=50 \mathrm{~m} / \mathrm{s}$ & Proposed & Ref [2] method & Ref [1] method \\
\hline Impact angle (deg) & -90.0148 & -90.4346 & -90.4618 \\
Error of impact angle (deg) & 0.0148 & 0.434 & 0.4618 \\
Miss distance (m) & 0.1413 & 0.1292 & 0.1692 \\
\hline
\end{tabular}

(b)

\begin{tabular}{lccc}
\hline$V_{T}=100 \mathrm{~m} / \mathrm{s}$ & Proposed & Ref [2] method & Ref [1] method \\
\hline Impact angle (deg) & -90.0025 & -92.9326 & -90.2880 \\
Error of impact angle (deg) & 0.0025 & 2.9326 & 0.2880 \\
Miss distance $(\mathrm{m})$ & 0.2488 & 0.2564 & 0.0408 \\
\hline
\end{tabular}

of autopilot is gained. The actual engagement is shown in Figure $6, V_{M}$ does not point to the virtual target, and $\sigma$ is the angle of line $M P$. If the error between $\gamma_{M}$ and $\sigma$ is elim- inated, the above guidance method based on virtual target is valid. To eliminate the error, the PID controller is a suitable choice. 
TABLE 8: Simulation conditions for Case IV.

\begin{tabular}{lcccccc}
\hline$r_{T}(\mathrm{~m})$ & $V_{T 0}(\mathrm{~m} / \mathrm{s})$ & $\gamma_{T 0}(\mathrm{deg})$ & $d V_{T}\left(\mathrm{~m} / \mathrm{s}^{2}\right)$ & $r_{M}(\mathrm{~m})$ & $V_{M}(\mathrm{~m} / \mathrm{s})$ & $\gamma_{M 0}(\mathrm{deg})$ \\
\hline$(5000,0)$ & 0 or 100 & 0 & 0.5 or -0.5 & $(0,0)$ & 250 & 30 \\
$a_{M}^{\max }\left(\mathrm{m} / \mathrm{s}^{2}\right)$ & $\xi$ & $t_{\tau}(\mathrm{s})$ & $\theta_{c}(\mathrm{deg})$ & & & \\
200 & 0.6 & 0.2 & -90 & & & \\
\hline
\end{tabular}
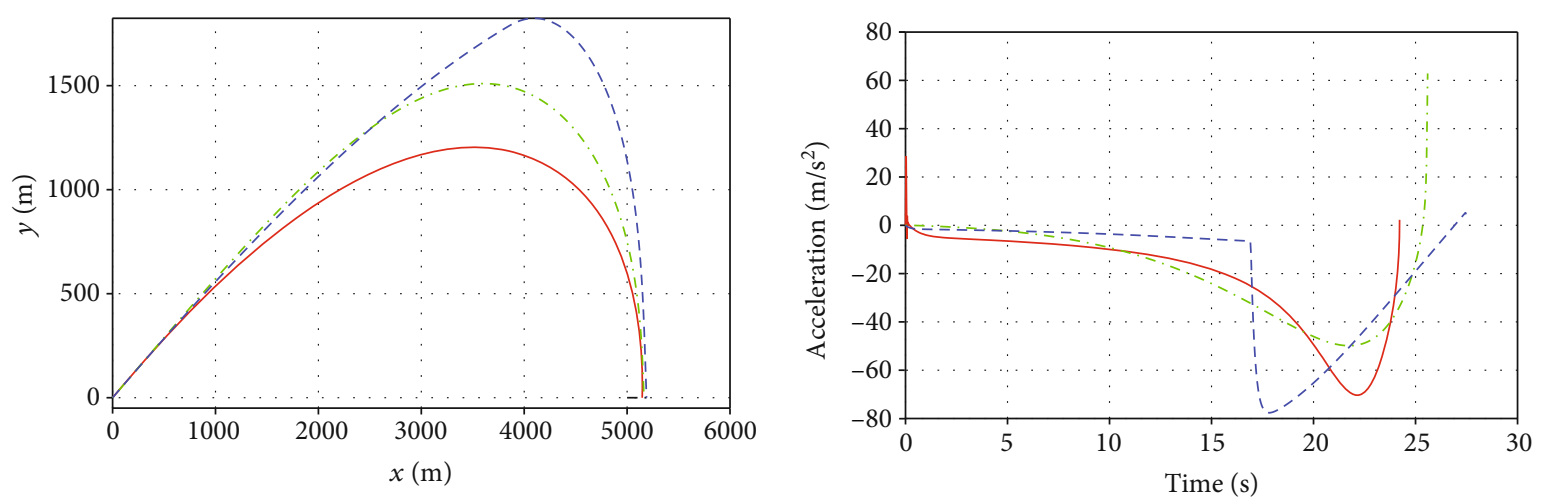

$\begin{array}{ll}\ldots \text { Target } & ---\operatorname{Ref}[2] \text { method } \\ \text { - Proposed } & -\cdot-\operatorname{Ref}[1] \text { method }\end{array}$

(a) Trajectories (target's speed up)

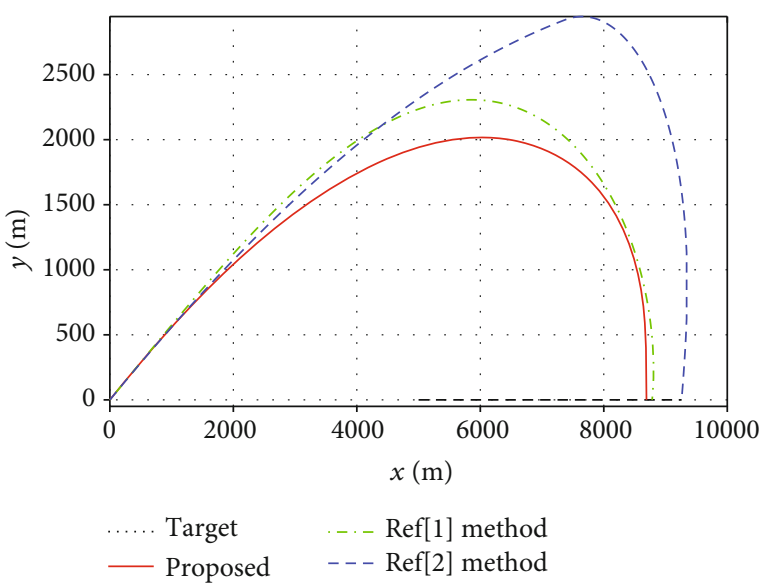

(c) Trajectories (target's speed down)
- Proposed

- - Ref[2] method

-. - Ref[1] method

(b) Acceleration (target's speed up)

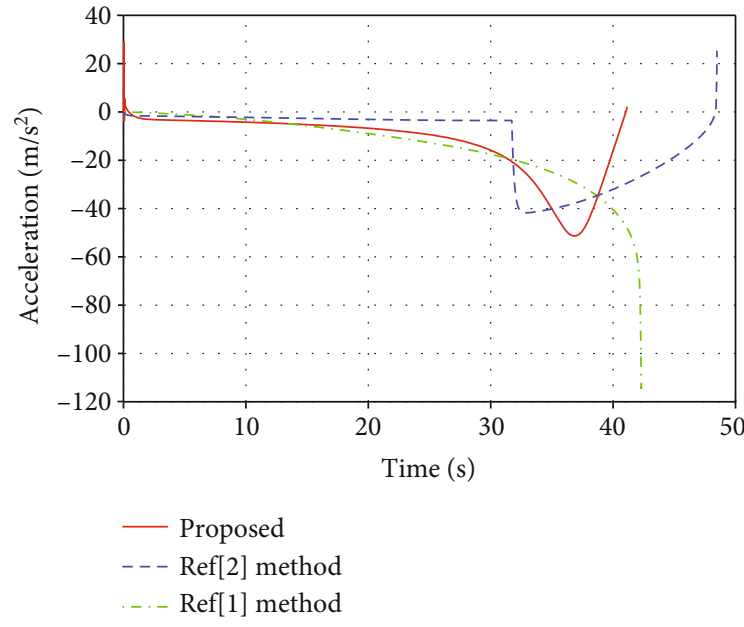

(d) Acceleration (target's speed down)

Figure 10: Case IV: variable velocity target.

$$
\begin{aligned}
& e=\sigma-\gamma_{M}, \\
& a=K_{P} e+K_{I} \int e d t+K_{D} \dot{e}, \\
& a_{M}= \begin{cases}a_{M}^{\max }, & \text { if } a>a_{M}^{\max }, \\
a, & \text { if }-a_{M}^{\max } \leq a \leq a_{M}^{\max }, \\
-a_{M}^{\max }, & \text { if } a<-a_{M}^{\max },\end{cases}
\end{aligned}
$$

where $K_{P}, K_{I}$, and $K_{D}$ are proportional gain, integral gain, and derivative gain, respectively. $a_{M}^{\max }$ is the maximum allowable lateral acceleration of the missile.

\section{Numerical Simulation}

In this section, a series of numerical simulations verify the effectiveness and advantages of the guidance method proposed by this paper, and some of which include comparisons with existing angle control guidance law.

4.1. Case I: All-Aspect Attack. In this subsection, the capacity of the all-aspect attack of the proposed guidance method will be verified by numerical simulations. Simulation conditions are shown in Table 2. The missile travels with a constant velocity, and $a_{M}^{\max }$ is the maximum lateral acceleration of 
TABLE 9: Simulation result for Case IV.

(a)

\begin{tabular}{lccc}
\hline Speed up & Proposed & Ref [2] method & Ref [1] method \\
\hline Impact angle (deg) & -90.0287 & -84.8451 & -85.8370 \\
Error of impact angle (deg) & 0.0287 & -5.1549 & -4.163 \\
Miss distance (m) & 0.1814 & 0.0671 & 0.2607 \\
\hline
\end{tabular}

(b)

\begin{tabular}{lccc}
\hline Speed down & Proposed & Ref [2] method & Ref [1] method \\
\hline Impact angle (deg) & -90.0035 & -103.0161 & -99.1056 \\
Error of impact angle (deg) & 0.0035 & 13.0161 & 9.1056 \\
Miss distance (m) & 0.0555 & 0.1514 & 0.1097 \\
\hline
\end{tabular}

the missile. $t_{\tau}$ is the time constant of missile's autopilot, and the target is stationary.

The simulation result is presented in Figure 7 and Table 3. The maximum miss distance is $0.2460 \mathrm{~m}$; it can be considered that all missiles hit the target. All impact angle errors are less than $0.553^{\circ}$, especially the errors of nonswitch methods are within $0.0097^{\circ}$; this can already be applied to most combat scenarios. It shows that the proposed guidance method can achieve a precise omnidirectional attack.

4.2. Compare with Other Impact Angle Control Guidance Methods. In this subsection, guidance methods proposed by $[1,2]$ are used as comparison methods.

4.2.1. Case II: Stationary Target. Three missiles employ the proposed method, literature [1] and literature [2] against a stationary target, respectively. Simulation conditions are shown in Table 4.

The simulation result is shown in Figure 8 and Table 5. When the target is stationary and the initial heading error is large, the three guidance methods have good guidance effects. Trajectories of the proposed method and the method in [1] are smooth, but the trajectory of the method in [2] has obvious signs of switching, which is also reflected in acceleration. The method proposed in this paper has the best impact angle control effect. If the initial heading error is small, performances of the guidance laws proposed by [2, 11] are significantly worse. This could be attributed to PNG that the methods in $[1,2]$ rely on. The formula of PNG is $a=N \dot{r} \dot{\lambda}$; when the initial heading error is small, $\dot{\lambda}$ is small, too. The lateral acceleration is too small to bend the trajectory enough, and the missile will not have a sufficiently large turning angle rate at the end of the engagement due to the limit of the lateral acceleration, so their missions failed. Figures 8 (b) and 8(d) show that acceleration of the proposed method converges to near 0 . The initial surge in acceleration is due to the initial velocity of the missile does not point to the virtual target, which shows that the initial heading error is essential to reduce the energy loss, although it did not affect the results here.
4.2.2. Case III: Constant Velocity Target. Replace the stationary target with a constant speed target, comparing the performances of the three guidance methods. Simulation conditions are shown in Table 6.

The simulation result is presented in Figure 9 and Table 7; three missiles all hit the target no matter the speed of the target is: $50 \mathrm{~m} / \mathrm{s}$ or $100 \mathrm{~m} / \mathrm{s}$. The angle errors of the three guidance methods are no more than $0.4618^{\circ}$ when $V_{T}=50 \mathrm{~m} / \mathrm{s}$; it can be considered that all the three guidance methods are successful to control the impact angle. But when $V_{T}=100 \mathrm{~m} / \mathrm{s}$, the angle error of the method proposed by [2] is $2.9326^{\circ}$; it failed to control the impact angle. Terminal acceleration of the method proposed in [1] is far more than 0 ; it is not conducive to building advantage. In the two numerical simulations, the proposed method based on virtual target has the least angle error, and its terminal acceleration converges to near 0 ; it has the best interception effect.

4.2.3. Case IV: Variable Velocity Target. In this subsection, variable velocity targets are considered. There are two kinds of variable speed targets, one with an initial speed of $0 \mathrm{~m} / \mathrm{s}$ and an acceleration of $0.5 \mathrm{~m} / \mathrm{s}^{2}$, and the other with an initial speed of $100 \mathrm{~m} / \mathrm{s}$ and an acceleration of $-0.5 \mathrm{~m} / \mathrm{s}^{2}$. Simulation conditions are presented in Table 8.

The simulation result is presented in Figure 10 and Table 9. Methods proposed by $[1,2]$ failed to control their impact angles. The missile employs that the proposed guidance method based on virtual target hits the variable speed target at the desired impact angle, no matter the target is accelerated or decelerated. It shows that this method has the best robustness.

\section{Conclusion}

A new guidance method based on the virtual target technique that allows a missile to impose the desired impact angle on the interception of a moving target has been presented in this paper. The position of the virtual target can be obtained by simple calculation of the states based on the missile and target, and thereby, the missile chases the virtual target by the pure pursuit guidance law to achieve better 
intercept performance. Simple geometric principles and Lyapunov stability theory are utilized to prove the strict convergence of the virtual target method. By the approach, $r$ and $a_{M}$ both converge to zero in the end, and the impact angle converges to $\theta_{c}$. These features guarantee the perfect interception on the target at the expected angle without saturation of terminal acceleration. Regarding the problem of omnidirectional attack, this paper proposes a two-stage guidance method, which is able to achieve an all-aspect precision strike. Numerical simulation proves that the proposed method has good performance against stationary or moving targets with constant or variable velocity, regardless of large or small initial heading error, and the guidance process is smooth with no chattering. It shows the proposed method is an excellent impact angle control method.

It must be pointed out that the value of $\xi$ can adjust the length of trajectory, so controlling the impact time by calculating the value of $\xi$ online is feasible. We will explore the algorithm of the value of $\xi$ to design an impact time and angle control guidance law.

\section{Data Availability}

The data used to support the findings of this study are included within the article.

\section{Conflicts of Interest}

The authors declare that they have no conflicts of interest.

\section{Acknowledgments}

This work was supported by the National Natural Science Foundation of China (Grant Nos. 61973182 and 61603210).

\section{References}

[1] A. Vairavan and A. Ratnoo, "A non-switching guidance law for impact angle constrained interception of moving targets," in AIAA Guidance, Navigation, and Control Conference, 2017, Grapevine, Texas, USA, 2017.

[2] A. Ratnoo and D. Ghose, "Impact angle constrained guidance against nonstationary nonmaneuvering targets," Journal of Guidance, Control, and Dynamics, vol. 33, no. 1, pp. 269275, 2010.

[3] A. Ratnoo, "Nonswitching guidance law for trajectory shaping control," Journal of Guidance, Control, and Dynamics, vol. 40, no. 10, pp. 2721-2728, 2017.

[4] M.-G. Seo, C.-H. Lee, and M.-J. Tahk, "New design methodology for impact angle control guidance for various missile and target motions," IEEE Transactions on Control Systems Technology, vol. 26, no. 6, pp. 2190-2197, 2018.

[5] B.-G. Park, H.-H. Kwon, Y.-H. Kim, and T.-H. Kim, "Composite guidance scheme for impact angle control against a nonmaneuvering moving target," Journal of Guidance, Control, and Dynamics, vol. 39, no. 5, pp. 1132-1139, 2016.

[6] X. Liu, Z. Shen, and P. Lu, "Closed-loop optimization of guidance gain for constrained impact," Journal of Guidance, Control, and Dynamics, vol. 40, no. 2, pp. 453-460, 2017.
[7] K. S. Erer and O. Merttopcuoglu, "Indirect impact-anglecontrol against stationary targets using biased pure proportional navigation," Journal of Guidance, Control, and Dynamics, vol. 35, no. 2, pp. 700-704, 2012.

[8] S. He, D. Lin, and J. Wang, "Integral global sliding mode guidance for impact angle control," IEEE Transactions on Aerospace and Electronic Systems, vol. 55, no. 4, pp. 1843-1849, 2019.

[9] Y. Zhao, Y. Sheng, and X. Liu, "Impact angle constrained guidance for all-aspect interception with function-based finite-time sliding mode control," Nonlinear Dynamics, vol. 85, no. 3, pp. 1791-1804, 2016.

[10] S. R. Kumar, S. Rao, and D. Ghose, "Nonsingular terminal sliding mode guidance with impact angle constraints," Journal of Guidance, Control, and Dynamics, vol. 37, no. 4, pp. 11141130, 2014.

[11] D. Cho, H. J. Kim, and M.-J. Tahk, "Impact angle constrained sliding mode guidance against maneuvering target with unknown acceleration," IEEE Transactions on Aerospace and Electronic Systems, vol. 51, no. 2, pp. 13101323, 2015.

[12] T. Shima, "Intercept-angle guidance," Journal of Guidance, Control, and Dynamics, vol. 34, no. 2, pp. 484-492, 2011.

[13] S. He, D. Lin, and J. Wang, "Robust terminal angle constraint guidance law with autopilot lag for intercepting maneuvering targets," Nonlinear Dynamics, vol. 81, no. 1-2, pp. 881-892, 2015.

[14] S. R. Kumar and D. Ghose, "Impact time and angle control guidance," in AIAA Guidance, Navigation, and Control Conference, Kissimmee, Florida, 2015.

[15] Z. Hou, L. Liu, and Y. Wang, "Time-to-go estimation for terminal sliding mode based impact angle constrained guidance," Aerospace Science and Technology, vol. 71, pp. 685694, 2017.

[16] Q. Hu, T. Han, and M. Xin, "New impact time and angle guidance strategy via virtual target approach," Journal of Guidance, Control, and Dynamics, vol. 41, no. 8, pp. 1755-1765, 2018.

[17] L. Sun, W. Wang, R. Yi, and S. Xiong, "A novel guidance law using fast terminal sliding mode control with impact angle constraints," ISA Transactions, vol. 64, pp. 12-23, 2016.

[18] Z. Chen, W. Chen, X. Liu, and J. Cheng, "Three-dimensional fixed-time robust cooperative guidance law for simultaneous attack with impact angle constraint," Aerospace Science and Technology, vol. 110, article 106523, 2021.

[19] S. Khankalantary and F. Sheikholeslam, "Robust extended state observer-based three dimensional integrated guidance and control design for interceptors with impact angle and input saturation constraints," ISA Transactions, vol. 104, pp. 299-309, 2020.

[20] C.-K. Ryoo, H. Cho, and M.-J. Tahk, "Optimal guidance laws with terminal impact angle constraint," Journal of Guidance, Control, and Dynamics, vol. 28, no. 4, pp. 724732, 2005.

[21] T. Tripathy and T. Shima, "Archimedean spiral-based intercept angle guidance," Journal of Guidance, Control, and Dynamics, vol. 42, no. 5, pp. 1105-1115, 2019.

[22] X. Yan, J. Zhu, M. Kuang, and X. Yuan, “A computationalgeometry-based 3-dimensional guidance law to control impact time and angle," Aerospace Science and Technology, vol. 98, article 105672, 2020. 
[23] Q. Chen, X. Wang, J. Yang, and Z. Wang, "Trajectory-following guidance based on a virtual target and an angle constraint," Aerospace Science and Technology, vol. 87, pp. 448$458,2019$.

[24] H. Shi, J. Zhu, M. Kuang, and X. Yuan, "Cooperative prediction guidance law in target-attacker-defender scenario," Science China Information Sciences, vol. 64, no. 4, pp. 1-3, 2021.

[25] R. V. Nanavati, S. R. Kumar, and A. Maity, "Lead-angle-based three-dimensional guidance for angle-constrained interception," Journal of Guidance, Control, and Dynamics, vol. 44, no. 1, pp. 190-199, 2021.

[26] I. Kim, H.-L. Choi, and I.-S. Jeon, “Closed-form impact-anglecontrol guidance of nose-dive missiles for maximum terminal speed," International Journal of Aeronautical and Space Sciences, vol. 22, no. 6, pp. 1428-1439, 2021. 